\title{
Positive symptoms and white matter microstructure in never-medicated first episode schizophrenia
}

\author{
V. Cheung ${ }^{1,3}$, C. P. Y. Chiu ${ }^{1}$, C. W. Law ${ }^{1}$, C. Cheung1, C. L. M. Hui ${ }^{1}$, K. K. S. Chan ${ }^{1}$, P. C. Sham ${ }^{1,2}$, \\ M. Y. Deng ${ }^{1,5}$, K. S. Tai ${ }^{4}$, P.-L. Khong ${ }^{4}$, G. M. McAlonan ${ }^{1,2 *}$, S.-E. Chua ${ }^{1,2}$ and E. Chen ${ }^{1,2}$ \\ ${ }^{1}$ Department of Psychiatry, University of Hong Kong, Pokfulam, SAR China \\ ${ }^{2}$ State Key Laboratory for Brain and Cognitive Sciences, University of Hong Kong, Pokfulam, SAR China \\ ${ }^{3}$ Department of Rehabilitation Sciences, The Hong Kong Polytechnic University, Hunghom, SAR China \\ ${ }^{4}$ Department of Diagnostic Radiology, Queen Mary Hospital, Pokfulam, SAR China \\ ${ }^{5}$ M.I.N.D. Institute, University of California, Davis, Medical Center, CA, USA
}

Background. We investigated cerebral structural connectivity and its relationship to symptoms in never-medicated individuals with first-onset schizophrenia using diffusion tensor imaging (DTI).

Method. We recruited subjects with first episode DSM-IV schizophrenia who had never been exposed to antipsychotic medication $(n=34)$ and age-matched healthy volunteers $(n=32)$. All subjects received DTI and structural magnetic resonance imaging scans. Patients' symptoms were assessed on the Positive and Negative Syndrome Scale. Voxel-based analysis was performed to investigate brain regions where fractional anisotropy (FA) values significantly correlated with symptom scores.

Results. In patients with first-episode schizophrenia, positive symptoms correlated positively with FA scores in white matter associated with the right frontal lobe, left anterior cingulate gyrus, left superior temporal gyrus, right middle temporal gyrus, right middle cingulate gyrus, and left cuneus. Importantly, FA in each of these regions was lower in patients than controls, but patients with more positive symptoms had FA values closer to controls. We found no significant correlations between FA and negative symptoms.

Conclusions. The newly-diagnosed, neuroleptic-naive patients had lower FA scores in the brain compared with controls. There was positive correlation between FA scores and positive symptoms scores in frontotemporal tracts, including left fronto-occipital fasciculus and left inferior longitudinal fasciculus. This implies that white matter dysintegrity is already present in the pre-treatment phase and that FA is likely to decrease after clinical treatment or symptom remission.

Received 26 April 2010; Revised 30 June 2010; Accepted 22 July 2010; First published online 1 September 2010

Key words: Diffusion tensor imaging, drug-naive, fractional anisotropy, PANSS, psychosis.

\section{Introduction}

Diffusion tensor imaging (DTI) is a novel research approach to assess the amount and preferred direction of diffusion of water molecules in vivo (Basser et al. 1994; Basser, 1995). In the brain, water molecules tend to diffuse along the direction of the axonal fibres wrapped by myelin sheathes; thus, DTI serves as a proxy measure of the orientation and structure of white matter comprising millions of axonal fibres grouped together in myelinated sheaths (Basser, 1995). Fractional anisotropy (FA) is a measure of the directional diffusion of water, values range from 0

* Address for correspondence: G. M. McAlonan, MD, PhD, Department of Psychiatry, The University of Hong Kong, Pokfulam, SAR China.

(Email: mcalonan@hku.hk) (completely anisotropic or randomly directed) to 1 (completely isotropic or uni-directional). FA scores increase in healthy children with better reading and spelling skills (Deutsch et al. 2005) and cognitive performance (Schmithorst et al. 2005) and decrease in adults with multiple sclerosis (Horsfield et al. 1998), traumatic brain injury (Gupta et al. 2005) and normal and abnormal ageing (Moseley, 2002). This makes it a useful tool to detect and quantify dynamic changes in white matter microstructure during neurodevelopment and degeneration of the brain. Since schizophrenia can be regarded as a disorder of neurodevelopment (Bullmore et al. 1997; Rapoport et al. 2005), DTI presents a safe and convenient tool to study how brain connectivity in patients differs from healthy individuals.

Using DTI, our group was the first to report widespread white matter disruption in individuals newly 
diagnosed with schizophrenia prior to treatment with antipsychotic medication (Cheung et al. 2008). We found that a range of white matter FA decreases adjacent to frontotemporal and striatolimbic regions in patients relative to healthy controls. Almost without exception, we and other groups have reported lower FA in patients compared with healthy controls (Kanaan et al. 2009). Most often implicated are the prefrontal and temporal lobes (Ardekani et al. 2003; Szeszko et al. 2005; Ellison-Wright \& Bullmore, 2009) and their interconnecting white matter tracts: uncinate fasciculus; cingulum bundle (Kubicki et al. 2003, 2005; Sun et al. 2003; Hubl et al. 2004; Zetzsche et al. 2007); arcuate fasciculus (Hubl et al. 2004; Kubicki et al. 2005); inferior longitudinal fasciulus (Ashtori et al. 2007) (for a review, see Kanaan et al. 2005, 2009; Kubicki et al. 2007; Kyriakopoulos et al. 2008). We also reported lower FA in the splenium of the corpus callosum (Cheung et al. 2008) and previous groups have also described reduced diffusivity in this principal interhemispheric tract (Foong et al. 2000, 2002; Ardekani et al. 2003; Hubl et al. 2004; Kalus et al. 2005) as well as in the striatum (Hubl et al. 2004; Kubicki et al. 2005, Szeszko et al. 2005) and the hippocampal/ parahippocampal region (Ardekani et al. 2003; Kalus et al. 2004, 2005).

Few studies have examined the relationship between white matter connectivity and symptomatology. Hubl et al. (2004) first explored this by comparing 13 chronically auditorily hallucinating patients with 13 non-auditorily hallucinating patients and 13 healthy controls. They reported significantly higher FA scores in the hallucinators versus the other two groups in the lateral parts of the temporo-parietal section of the arcuate fasciculus and in parts of the anterior corpus callosum. The lateral part of the arcuate fasciculus links Broca's area in the frontal lobe to Wernicke's area in the temporal lobe and is disrupted in aphasia. The hallucinating group also had greater FA than the non-hallucinating group in the left cingulum bundle, which may represent aberrant emotional circuitry. Others have failed to replicate these results. Seok and co-workers, in a group of likewise intractably hallucinated patients as compared with non-hallucinators and healthy controls, found only a positive correlation between severity of auditory hallucinations and FA score in the left frontal part of the superior longitudinal fasciculus (Seok et al. 2007). Fujiwara and co-workers also used DTI based on a region-of-interest analysis restricted to the anterior and posterior cingulate bundles and found that positive symptoms in chronically psychotic patients were positively correlated with FA scores in the posterior cingulate bundle (Fujiwara et al. 2007). In contrast, Skelly and co-workers used voxel-based
DTI to show lower FA in 25 patients with chronic schizophrenia compared with 25 healthy controls. They reported that positive symptoms were inversely correlated with FA scores in diverse regions, including the left uncinate fasciculus, right sagittal striatum and left superior longitudinal fasciculus (Skelly et al. 2008).

Thus, there is discrepancy in the literature and it may be useful to clarify the direction of the relationship between white matter FA and symptom severity using a first-episode sample. A handful of DTI studies have recruited such patients (Price et al. 2007a, b; Cheung et al. 2008; Friedman et al. 2008; Peters et al. 2008) and while there have been negative findings (Friedman et al. 2008; Peters et al. 2008), others noted deep frontotemporal white matter connections (Kyriakopoulos et al. 2008), especially the inferior fronto-occipital fasciculus (Ellison-Wright \& Bullmore, 2009), uncinate fasciculus (Price et al. 2007b) as well as callosum (Price et al. 2007a ; Kyriakopoulos \& Frangou, 2009) and cerebellar tracts (Kyriakopoulos \& Frangou, 2009). These findings appear weaker than those described above in chronic populations, in which dose-dependent FA increase may have contributed (Okugawa et al. 2004; Shergill et al. 2007). The tendency to step up medication afflicts chronically relapsing samples and this in turn can confound the interpretation of results (Kanaan et al. 2005; Kyriakopoulos et al. 2008). In addition, the issue of sample size and heterogeneity in data processing (Zetzsche et al. 2007; Ellison-Wright \& Bullmore, 2009) may have contributed to inconsistencies across studies. For example, antipsychotic medication can increase basal ganglia volume (Chakos et al. 1994; Sigmundsson et al. 2001; Chua et al. 2008; Deng et al. 2009; Leung et al. 2009) and even just a few days of antipsychotic treatment can modulate FA (Kanaan et al. 2009). Therefore, we conceived a study to address the role of symptoms on white matter organization in a voxel-by-voxel basis in patients presenting for the first time with schizophrenia, who had never previously been exposed to antipsychotic medication. The aim of the study is to address the issues of chronicity and medication exposure as potential confounders. We sought to limit their effect by ensuring that our sample consists of individuals newly diagnosed with schizophrenia presenting in their first episode of illness and all neuroleptic-naive.

\section{Method}

\section{Subjects}

All subjects recruited were aged between 18 and 45 years. They had 6-18 years of education, were 
Chinese and had given fully informed written consent to participate in a study approved by the Hong Kong West Cluster Institutional Review Board of the hospital. Subjects were excluded if they had a history of neurological illness (such as epilepsy, strokes or meningitis), head injury that required hospitalization, persistent headaches, genetic disorder (such as tuberous sclerosis, fragile $X$ syndrome), electroconvulsive therapy, history of drug and/or alcohol abuse, hyperactivity, mental retardation, suspension from school for $>6$ months. They were also excluded if they had taken medication for any chronic medical condition in the previous week.

Patients were recruited from the teaching hospital of The University of Hong Kong. Inclusion criteria were no previous antipsychotic treatment, first experience of psychotic symptoms (i.e. hallucinations, and/or delusions and/or thought disorder), no significant mood/organic disorder, with decline in daily functioning and fulfilling criteria for a diagnosis of DSM-IV schizophrenia (APA, 1994) after assessments by specialist clinician investigators in psychiatry (C.P.Y.C., C.W.L.). Healthy controls from the local community were recruited by advertisement.

Patients were screened on the first day of presentation to hospital and gave full informed written consent to participate. Symptoms were rated by a specialist psychiatrist with clinical responsibility for the care of the patients using the Positive and Negative Syndrome Scale (PANSS; Kay et al. 1987, 1988), with intra-class correlation coefficients $0.73-0.83$ (Chen et al. 2005) based on a 30-item scale with positive, negative and general psychopathology items demonstrating high reliability and validity (Kay et al. 1988; Kay, 1990). A magnetic resonance imaging (MRI) scan was performed according to the protocol below before initiating antipsychotic medication.

\section{MRI data acquisition}

DTI and T2/PD datasets were acquired by GE Signa 1.5 T system (General Electric, USA). A 5-min DTI sequence followed the protocol of Leung et al. (2004) and comprised single-shot spin-echo echo-planar imaging with $\mathrm{TR}=10000 \mathrm{~ms}, \mathrm{TE}=100 \mathrm{~ms}$, acquisition matrix = $128 \times 128$, and field of view $=28 \mathrm{~cm}$, slice thickness of 5 - with $1.5 \mathrm{~mm}$ gap. In total, 25 volumes had diffusionsensitizing gradient encoding (Basser et al. 1994; Basser, 1995) applied along 25 directions with a diffusion-weighted factor $b=1200 \mathrm{~s} / \mathrm{mm}^{2}$ and one volume, $B_{0}$, without a diffusion gradient, $b=0 \mathrm{~s} / \mathrm{mm}^{2}$ (see Cheung et al. 2008). A dual-echo fast spin echo sequence provided T2/PD datasets aligned to AC-PC across the whole brain, $3 \mathrm{~mm}$ thick, Nex $=0$, TR 5-6 s,
TE $20 / 80$ ms, matrix $256 \times 192$ (see McAlonan et al. 2005).

\section{Imaging processing and analysis}

Image pre-processing was performed using SPM2 (Wellcome Department of Cognitive Neurology, Institute of Neurology, UK) and the Diffusion II toolbox (http://sourceforge.net/projects/spmtools) running in MATLAB 6.5 (The MathWorks, Inc., USA). The DTI pre-processing sequence was based upon the optimized voxel-based morphometric approach of Good et al. (2001) and Cheung et al. (2008).

To examine the relationship between symptoms and FA, whole brain FA maps of the patient group were correlated separately with positive and negative PANSS scores in a voxel-by-voxel manner using a standard linear regression model (Genovese et al. 2002) with results thresholded at a false discovery rate (FDR) of $p<0.05$ (two tailed, corrected). As in our previous study (Cheung et al. 2008), we used the FDR threshold of $p<0.05$ (two tailed, corrected), where FDR is the proportion of false positives (incorrect rejections of the null hypothesis) among those tests for which the null hypothesis is rejected (Genovese et al. 2002). We relied upon the FDR method to control for multiple comparisons leading to type 1 error under the null hypothesis (Bullmore et al. 1999) as opposed to the Bonferroni correction, which is regarded as overly strict, where neighbouring pixels are interdependent (Genovese et al. 2002). All MNI coordinates were converted to Talairach using a standard non-linear algorithm (Brett, 2002).

\section{Results}

Subjects with schizophrenia $(n=34)$ had a mean age of 25.4 (s.D. =7.5) years. There were 17 females, who were all right-handed. They had a PANSS positive symptomatology mean score of 19.9 (S.D. =4.2), PANSS negative symptomatology mean score of 15.0 (S.D. $=$ 7.2). The healthy controls had a mean age of 27.6 (s.D. $=8.5$ ) years and did not differ significantly from the patients $(p>0.03), 15$ were female and all were right-handed and did not differ significantly with the patients $(p=0.9)$. In this study, all subjects were righthanded and are extended from a previous sample (25 patients, 26 healthy controls) from Cheung et al. (2008).

\section{Voxel-wise correlation of FA scores and PANSS}

In total, 34 never-medicated patients with first-episode schizophrenia and 32 healthy controls successfully underwent a MRI scan. Altogether, 31 patients with 
never-medicated first-episode schizophrenia who had PANSS data for positive and negative PANSS scores were entered into the analysis. We then compared all 34 patients with the 32 healthy controls on mean FA values for 11 clusters that showed significant correlation with PANSS scores (Table 1).

We found that FA values in 11 frontal (Figs 1 and 2) and temporal-limbic (Figs 1 and 3) clusters were positively associated with high positive symptom scores [voxel level $p<0.05$ (FDR-corrected), cluster size 100, cluster level $p<0.05$ (uncorrected)]. Notwithstanding this, for all 11 clusters, the FA values were lower in patients than in healthy controls, significantly so around the left anterior cingulate and superior temporal regions (see Table 1). Thus, the closer FA values in the patient group were to control measures, the greater the number of positive symptoms. There were no brain regions showing negative correlation with positive PANSS. There were also no brain regions showing any correlation with negative PANSS.

\section{Discussion}

Our principal finding is that in antipsychotic-naive patients newly diagnosed with schizophrenia, positive symptoms showed a significant positive correlation with widespread regions, including fronto-occipital and temporo-limbic white matter FA scores. Specifically, these regions included the right frontal lobe, left anterior cingulate gyrus, left body of cingulum, left superior temporal gyrus, right middle temporal gyrus, right inferior parietal gyrus, left cuneus, left genu of corpus callosum, right extension of posterior internal capsule. However, in all of these regions FA scores were lower in patients than healthy controls. Our present study extends our previous DTI work on never-medicated patients in their first episode of schizophrenia (Cheung et al. 2008), indicating that patients with FA values approaching control levels in fronto-occipital and temporo-limbic tracts have the greatest number of positive symptoms.

In our previous study (Cheung et al. 2008), we reported decreased FA in patients compared with healthy controls in a widespread pattern, which included the fronto-occipital and temporo-limbic regions corresponding to the left fronto-occipital fasciculus and inferior longitudinal fasciculus, white matter adjacent to right precuneus, splenium of corpus callosum, right posterior limb of internal capsule, white matter adjacent to right substantia nigra and left cerebral peduncle. The current study found that, despite lower FA in the patient group relative to controls, there was a positive correlation between positive symptoms and FA encompassing a widespread network including frontooccipital and temporo-limbic pathways. This result may be considered rather counter-intuitive in that more florid positive symptoms of schizophrenia are associated with white matter FA values very similar to control levels. We note that FA relatively increased with positive symptoms even though, overall, the absolute FA scores were all lower in the group with schizophrenia.

Widespread cortical thinning has been described both in first-episode schizophrenia in the frontopolar, cingulate and occipital regions (Narr et al. 2005). The same group had earlier reported that very early onset individuals followed up over 5 years showed striking grey matter loss originating in the parietal cortex dynamically moving to temporal then frontal cortices (Thompson et al. 2001). They noted that this grey matter loss correlated with psychotic symptom severity as well as sensorimotor (including visuo-spatial and auditory functions) and executive function impairments (Thompson et al. 2001). Cortical thinning is believed to reflect underlying reduced neuropil and neuronal size with alterations in synaptic, dendritic and axonal organization (Harrison, 1999) and, if progressive, may imply that dyconnectivity gets worse over time. Hence, our data in newly diagnosed and never-medicated subjects with schizophrenia support the position that there is already widespread cortical dysconnectivity with lower FA scores in patients compared with controls across the board.

When we examined the overlap in spatial distribution of 'signature' between our previous study (Cheung et al. 2008) and the current one, we noted that three regions in the present study overlapped with the previous one, in which seven regions had been reported. All showed significantly reduced FA in patients as compared with controls. These three regions in the present study are left anterior cingulate gyrus (anterior to fronto-occipital fasciculus), left superior temporal gyrus (adjacent to inferior longitudinal fasciculus) and right extension from posterior limb of internal capsule. Since these regions are dopamine-rich, we believe that they resonate with the propensity for antipsychotics to modulate positive symptoms and may be evidence of state- rather than trait-related dysconnectivity. Hubl et al. (2004) had reported higher white matter directionality in the temporo-parietal region in auditorily hallucinated patients versus non-auditorily hallucinated controls. Other studies have also reported findings in this direction (Fujiwara et al. 2007; Seok et al. 2007), although they are not directly comparable to ours since all assessed chronically ill patients with persistent symptoms and possibly lengthy exposure to antipsychotics. Our findings are in keeping with those from three recent DTI studies, which have also reported a positive correlation between positive symptoms and FA. 
Table 1. White matter fractional anisotropy $(F A)$ differences in patients compared with healthy controls

\begin{tabular}{|c|c|c|c|c|c|c|c|c|c|}
\hline \multirow{2}{*}{$\begin{array}{l}\text { Brain regions }{ }^{\mathrm{a}} \text { showing significant correlation } \\
\text { with symptoms }\end{array}$} & \multirow{2}{*}{$\begin{array}{l}\text { Cluster } \\
p \text { value }\end{array}$} & \multirow{2}{*}{$\begin{array}{l}\text { Cluster size } \\
\text { (voxel) }\end{array}$} & \multicolumn{3}{|c|}{ Talairach coordinates $(\mathrm{mm})$} & \multirow{2}{*}{$\begin{array}{l}\text { Voxel } \\
\text { Z value }\end{array}$} & \multicolumn{2}{|l|}{ FA mean (S.D.) } & \\
\hline & & & $x$ & $\mathrm{y}$ & $\mathrm{z}$ & & Control $(n=32)$ & Patient $(n=34)$ & \\
\hline \multicolumn{10}{|l|}{ Positive correlation with positive PANSS scores } \\
\hline $\begin{array}{l}\text { R pre-central sulcus } \\
\text { (above arcuate/superior longitudinal fasiculus) }\end{array}$ & 0.028 & 400 & 34 & 5 & 27 & 4.55 & $0.2988(0.029)$ & $0.2929(0.037)$ & N.S. \\
\hline $\begin{array}{l}\mathrm{R} \text { post-central gyrus } \\
\text { (adjacent to fronto-occipital fasciculus) }\end{array}$ & $<0.0005$ & 1770 & 23 & 35 & 16 & 4.47 & $0.3250(0.025)$ & $0.3132(0.038)$ & N.S. \\
\hline $\begin{array}{l}\mathrm{R} \text { inferior frontal gyrus } \\
\text { (fronto-occipital fasciculus) }\end{array}$ & 0.016 & 751 & 16 & 30 & 1 & 3.78 & $0.4312(0.036)$ & $0.4187(0.048)$ & N.S. \\
\hline $\begin{array}{l}\mathrm{L} \text { body of cingulum } \\
\text { (adjacent to fronto-occipital fasciculus) }\end{array}$ & 0.001 & 1114 & -15 & 2 & 29 & 4.45 & $0.3667(0.030)$ & $0.3635(0.053)$ & N.S. \\
\hline $\begin{array}{l}\mathrm{L} \text { anterior cingulate gyrus } \\
\text { (anterior to fronto-occipital fasciculus) }\end{array}$ & 0.004 & 784 & -19 & 38 & 12 & 4.02 & $0.3699(0.031)$ & $0.3398(0.041)$ & * \\
\hline \multicolumn{10}{|l|}{ Temporo-parietal } \\
\hline $\begin{array}{l}\mathrm{R} \text { middle temporal gyrus } \\
\text { (adjacent to inferior longitudinal fasciculus) }\end{array}$ & 0.007 & 649 & 45 & -50 & 4 & 4.05 & $0.3641(0.051)$ & $0.3421(0.062)$ & N.S. \\
\hline $\begin{array}{l}\text { L superior temporal gyrus } \\
\text { (adjacent to inferior longitudinal fasciculus) }\end{array}$ & 0.016 & 498 & -38 & -33 & 6 & 3.63 & $0.3699(0.050)$ & $0.3398(0.058)$ & $* *$ \\
\hline $\begin{array}{l}\mathrm{R} \text { inferior parietal gyrus } \\
\text { Occipital }\end{array}$ & 0.038 & 349 & 29 & -31 & 37 & 3.53 & $0.3662(0.030)$ & $0.3584(0.052)$ & N.S. \\
\hline $\begin{array}{l}\text { L cuneus } \\
\text { Subcortical }\end{array}$ & 0.006 & 703 & -19 & -75 & 16 & 3.99 & $0.3117(0.025)$ & $0.2987(0.047)$ & N.S. \\
\hline L genu of corpus callosum & 0.030 & 392 & -5 & 18 & 21 & 3.92 & $0.3551(0.041)$ & $0.3477(0.057)$ & N.S. \\
\hline $\begin{array}{l}\text { R extension from posterior internal capsule to central } \\
\text { sulcus, along fronto-occipito fasciculus }\end{array}$ & 0.010 & 594 & 18 & -5 & 22 & 4.61 & $0.3617(0.039)$ & $0.3447(0.052)$ & N.S. \\
\hline
\end{tabular}

PANSS, Positive and Negative Syndrome Scale; R, right; L, left; N.s., non-significant group difference in mean FA.

${ }^{a}$ Brain regional labels are suggested for guidance only.

${ }^{\mathrm{b}} p<0.05$ (false discovery rate-corrected).

* Significant group difference in mean FA; $p<0.05$, uncorrected.

** Significant group difference in mean FA; $p<0.005$, uncorrected. 


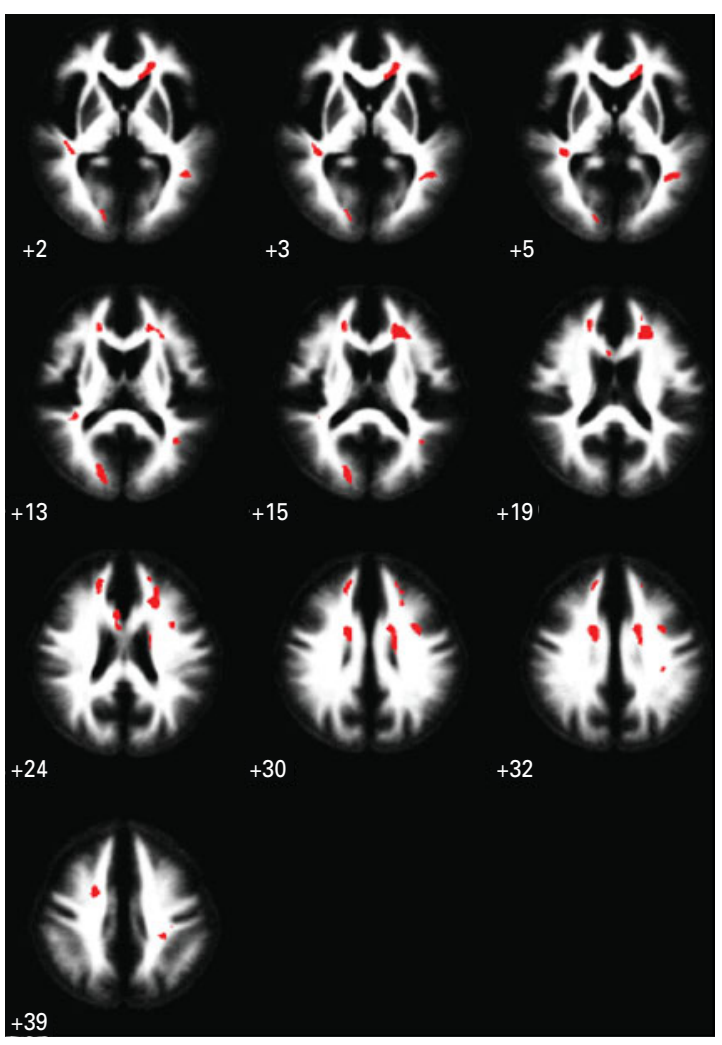

Fig. 1. Functional anisotropy (FA) score correlates of positive symptoms in schizophrenia. Red clusters show significant positive correlations of white matter FA with positive symptom scores in never-medicated patients with first-episode schizophrenia $(n=31)$. There were no significant negative correlations of white matter FA with negative symptom scores. The maps are orientated with the right side of the brain shown on the right side of the panel. The $z$ coordinate for each axial slice in the standard space of Talairach and Tournoux is given in $\mathrm{mm}$.

Delusions/hallucinations positively correlated with FA in the left inferior fronto-occipital fasciculus (Szeszko et al. 2008). A positive correlation was also noted between delusions of passivity and FA in the frontocingulate region (Sim et al. 2009) and between auditory hallucinations with FA in the arcuate/ superior longitudinal fasciculi and anterior cingulum (Shergill et al. 2007). These sites of white matter dysconnectivity are extensive (for review, see Catani et al. 2002), comprising the inferior fronto-occipital tract (connects the frontal cortex with posterior temporal and occipital cortex), the superior longitudinal fasculus (links the frontal with parietal and temporal cortices, the cingulum (joins the inferior temporal pole with the subrostral frontal lobe, cingulate gyrus and genu of corpus callosum). We believe that this myriad of white matter deficits is in keeping with evidence in the literature of established cortical thinning in first-episode, minimally treated (Narr et al. 2005), as well as very early onset, schizophrenia (Thompson et al. 2001). Conceivably, some discrepancy in the extant literature may arise from sample characteristics, such as effects of medication and chronicity, since these are difficult to disentangle from pathology. For example, of the three studies cited above, the Szeszko group included the youngest patients (mean age $=25$ years), who were newly diagnosed but many already in receipt of medication. They noted that medication effects are still not well understood but that FA scores inversely correlated with the duration of antipsychotic treatment in the left superior longitudinal fasciculus (Szeszko et al. 2008). Our study was conducted with neuroleptic-naive individuals and our findings, taken with these recent studies, argue for the presence of frontotemporo-limbic dysconnectivity. This parsimonious interpretation is also propounded in a recent meta-analysis by Ellison-Wright \& Bullmore (2009). However, for patients on treatment it is worth bearing in mind the possible contribution from neuroleptic treatment, since there is a duration- (Szeszko et al. 2008) and dosage- (Okugawa et al. 2004) dependent relationship between antipsychotic treatment and FA score. Our finding that positive symptoms are positively correlated with FA appears at odds with the study by Skelly et al. (2008). The latter group reported that chronically treated patients (mean age $=34$ years) had lower brain FA in association tracts (including left superior longitudinal fasciculus), which inversely correlated with positive symptoms. This discrepancy with our own results deserves to be carefully addressed. One possibility is that younger patients with minimal or no exposure to medication experience FA lowering further over time, i.e. an inverse relationship with positive symptoms may emerge as the illness progresses or as a consequence of medication exposure (Szeszko et al. 2008). However, as our study was cross-sectional, we anticipate that a future longitudinal follow-up of our patients would be an ideal approach to try to reconcile results from samples of patients at different stages of illness.

Our cross-sectional analysis cannot speak to 'causal' mechanisms but does prompt some interpretation. Mild variation in FA may not reflect serious pathology in neurotypical individuals but here potentially reveals vulnerability in those with psychosis. For example, positive symptoms could be generated via aberrant activation of white matter pathways, which remain reasonably 'intact'. Classically, symptoms in schizophrenia aggregate into distinct symptom complexes, suggesting that a distinct neural substrate can be investigated by neuroimaging (Buchanan \& Carpenter, 1997). 'Positive' symptoms (hallucinations, delusions, thought disorder) are thought to reflect 


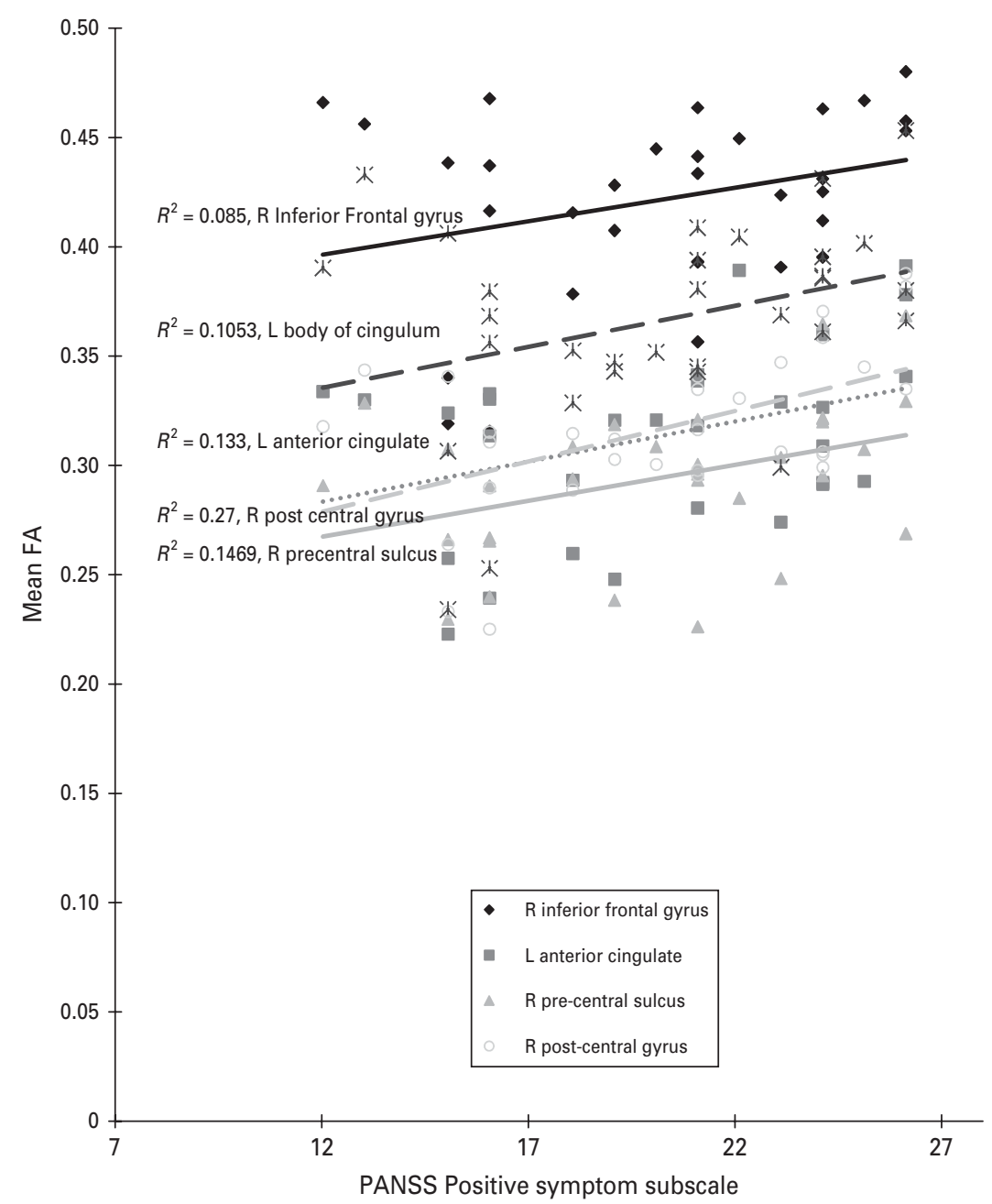

Fig. 2. Scatter plot of the relationship between functional anisotropy (FA) of significant frontal clusters with positive symptom subscale on the Positive and Negative Syndrome Scale (PANSS). A best-fitted least square line was plot for each of the five frontal areas and their corresponding $R^{2}$ given next to each of the best-fitted lines.

'state' and are more treatment-responsive (Buchanan \& Carpenter, 1997), 'negative' symptoms represent 'trait' (Buchanan \& Carpenter, 1997) and are regarded as having a likely brain structural basis (Crow, 1985). Persistently untreated positive (Flaum et al. 1995; Sumich et al. 2005) or negative (Chua et al. 1997; Wible et al. 2001) symptoms may well increase the likelihood of brain structural abnormality but, at first episode, the extent of white matter pathophysiology is still limited.

We cautiously suggest that our results indicate that such limited brain structural pathology at first presentation might provide a substrate for expression of positive symptoms, characteristic of the early phase of illness. There is some evidence to support this in the literature. For example, the duration of untreated illness is associated with worse prognosis (Barnes et al. 2008) and more extensive brain structural differences (Lappin et al. 2006). Positive symptoms are less prominent over time (Arndt et al. 1995) and, with each relapse, prognosis worsens and treatment dosages often escalate (Lieberman et al. 1993). Our data concur with others (Hubl et al. 2004; Fujiwara et al. 2007; Seok et al. 2007), which imply that disease progression and symptomatology are related to changes in the organization of white matter circuitry. The corollary of our finding of a positive correlation between FA scores and positive symptoms in the left fronto-occipital and temporo-limbic tracts is that, over time (as symptoms abate with treatment), FA may decline and this can represent a marker for treatment or prognosis. Addressing this question in a longitudinal follow-up study may help to determine the extent to which positive and negative symptoms reflect white matter pathology and whether FA can serve as a marker for treatment or prognosis. Perhaps one way forward would be to evaluate future treatment outcome based on symptoms and/or functioning at 1 year or 2, and then to enter this as covariate into the baseline scan in 


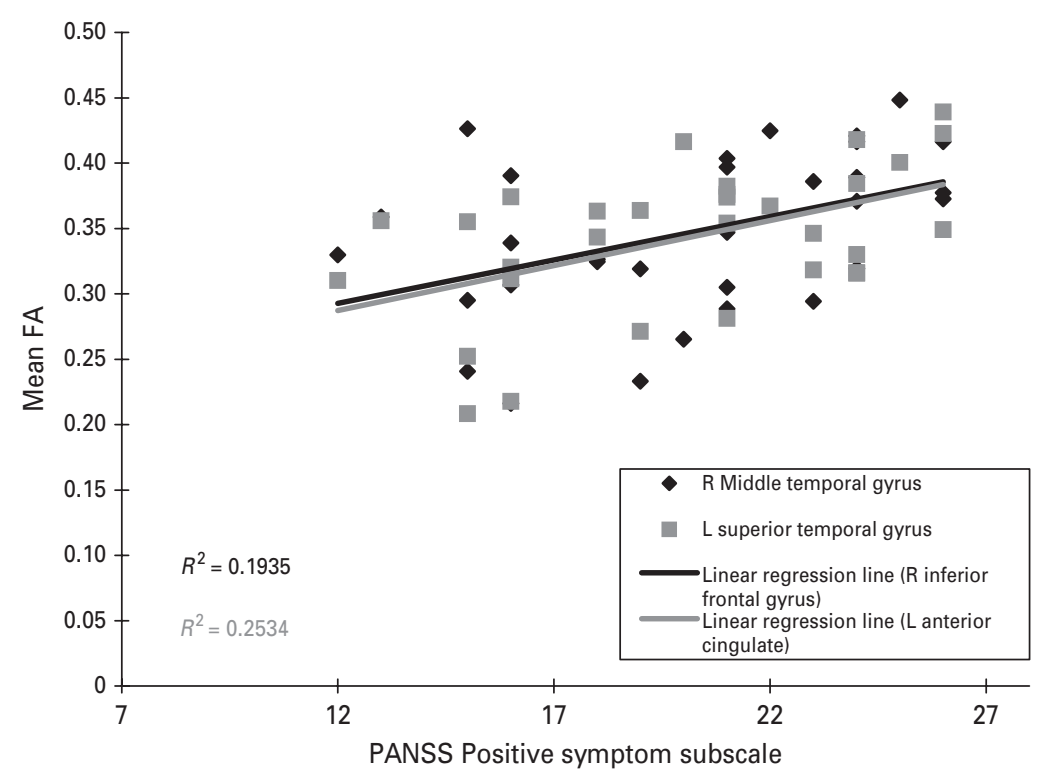

Fig. 3. Scatter plot of the relationship between functional anisotropy (FA) of significant temporal clusters with positive symptom subscale on the Positive and Negative Syndrome Scale (PANSS). A best-fitted least square line was plotted for each of the five frontal areas and their corresponding $R^{2}$ given next to each of the best-fitted lines.

order to determine if baseline FA score may be used to help predict future outcome.

The brain pathology in schizophrenia is suggested to arise from dysregulation of the normal dendritic organization that accompanies a critical surge in white matter growth from adolescence to early adulthood (Sowell et al. 1999), when prodrome symptoms typically begin. Harrison (1999) has argued cogently that key susceptibility genes (such as catechol-O-methyltransferase, neuregulin, dysbindin) converge toward the risk of schizophrenia by their role in synaptic plasticity/function (Frankle et al. 2003) so as to impair higher order information processing. In other words, this disorder appears, in its fundamental form, to be one of impaired stabilization and development of cortical micro-circuitry (Harrison, 1999; Winterer and Weinberger, 2004). Consequently, the directionality of neuroimaging findings in schizophrenia may be quite difficult to predict, but DTI evidence for disruption of white matter connections linked to a multiplicity of symptoms fits with asynchronization of neural circuitry during neurodevelopment, resulting in synaptic misconnections (Andreasen et al. 1999; Frankle et al. 2003; Whitefield-Gabrieli et al. 2009).

\section{Strengths and weaknesses of study}

The study involved a never-medicated, first-episode group of patients with schizophrenia and aimed to investigate how their symptoms are related to system of white matter connectivity. It employed a voxelbased approach not constrained by prior hypotheses.
Patients with schizophrenia were carefully diagnosed and ethnically homogeneous and well balanced with the healthy controls. Although missing data meant that we were not able to enter the duration of untreated psychosis as a covariate to explore the possibility of a relationship between duration of psychosis and FA score, we observed that the duration of psychosis for many subjects was approximately 1 year. This is a potential limitation of the study, together with the DTI sequence used, which could be improved in power with thinner slices and a more powerful magnet, which, fortunately, will become accessible to our patients for future studies.

A between-group analysis followed by exploration of symptom correlates of regions showing significant differences would arguably have been the statistically more powerful and conventional approach. However, we previously published cross-sectional data on a sample including many of the patients and controls from the present study (Cheung et al. 2008). We considered that the regions uncovered in a cross-sectional study might encompass aspects of the illness not exclusively pertaining to symptoms, such as predisposition/vulnerability to disorder. Therefore, since the main thrust of the current study was to examine the symptom-FA interaction, we decided to perform whole-brain correlational analysis. As discussed earlier, the FA correlates of positive symptoms coincided with fewer than half the regions of group difference identified in the cross-sectional analysis. We interpret this finding as pointing towards a difference in the white matter pathways contributing to more 'static' 
disease traits responsible for group differences and those that are involved in generation of positive symptoms.

In conclusion, fronto-occipital and temporo-limbic white matter connectivity positively correlates with positive symptom scores in first-episode schizophrenia. These effects on white matter connections are not influenced by pharmacotherapy and therefore appear to be disease-related. Future work will focus on mapping the progression of illness using FA, together with other neuroimaging markers, to investigate how the administration of antipsychotic agents can modify brain structure and symptoms.

\section{Acknowledgements}

This work was supported by a research grant to Dr S. E. Chua and Dr E. Y. H. Chen from the CRCG, The University of Hong Kong.

\section{Declaration of Interest}

None.

\section{References}

Andreasen NC, Nopoulos P, O'Leary DS, Miller DD, Wassink T, Flaum M (1999). Defining the phenotype of schizophrenia, cognitive dysmetria and its neural mechanisms. Biological Psychiatry 46, 908-920.

APA (1994). Diagnostic and Statistical Manual of Mental Disorders, 4th edn. American Psychiatric Press:

Washington DC.

Ardekani BA, Nierenberg J, Hoptman MJ, Javitt DC, Lim KO (2003). MRI study of white matter diffusion anisotropy in schizophrenia. Neuroreport 14, 2025-2029.

Arndt S, Andreasen NC, Flaum M, Miller D, Nopoulos P (1995). A longitudinal study of symptom dimensions in schizophrenia, prediction and patterns of change. Archives of General Psychiatry 52, 352-360.

Ashtori M, Cottone J, Ardekani B, Cervellione K, Szeszko PR, Wu J, Chen S, Kumra S (2007). Disruption of white matter integrity in the inferior longitudinal fasciculus in adolescents with schizophrenia as revealed by fiber tractography. Archives of General Psychiatry 64, 1270-1280.

Barnes TR, Leeson VC, Mutsatsa SH, Watt HC, Hutton SB, Joyce EM (2008). Duration of untreated psychosis and social function, 1-year follow-up study of first-episode schizophrenia. British Journal of Psychiatry 193, 203-209.

Basser PJ (1995). Inferring microstructural features and the physiological state of tissues from diffusion-weighted images. NMR in Biomedicine 8, 333-344.

Basser PJ, Mattiello J, LeBihan D (1994). MR diffusion tensor spectroscopy and imaging. Biophysics Journal 66, 259-267. Brett M (2002). The MNI Brain and the Talairach Atlas. MRC Cognition and Brain Sciences Unit: Cambridge.
Buchanan RW, Carpenter WT (1997). The neuroanatomies of schizophrenia. Schizophrenia Bulletin 23, 367-372.

Bullmore ET, Frangou S, Murray RM (1997). The dysplastic net hypothesis, an integration of developmental and dysconnectivity theories of schizophrenia. Schizophrenia Research 28, 143-156.

Bullmore ET, Suckling J, Overmeyer S, Rabe-Hesketh S, Taylor E, Brammer MJ (1999). Global, voxel, and cluster tests, by theory and permutation, for a difference between two groups of structural MR images of the brain. IEEE Transactions on Medical Imaging 18, 32-42.

Catani M, Howard RJ, Pajevic S, Jones DK (2002). Virtual in vivo interactive dissection of white matter fasciculi in the human brain. Neuroimage 17, 77-94.

Chakos MH, Lieberman JA, Bilder RM, Borenstein M, Lerner G, Bogerts B, Wu H, Kinon B, Ashtari M (1994). Increase in caudate nuclei volumes of first-episode schizophrenic patients taking antipsychotic drugs. American Journal of Psychiatry 151, 1430-1436.

Chen EYH, Hui CL, Dunn EL, Miao MYK, Yeung WS, Wong CK, Chan WF, Tang WN (2005). A prospective 3-year longitudinal study of cognitive predictors of relapse in first-episode schizophrenic patients. Schizophrenia Research 77, 99-104.

Cheung V, Cheung C, McAlonan GM, Deng MY, Wong JGWS, Yip L, Tai KS, Khong PL, Sham PC, Chua SE (2008). A DTI study of structural dysconnectivity in never-medicated, first-episode schizophrenia. Psychological Medicine 38, 877-885.

Chua SE, Deng MY, Chen EYH, Law CW, Chiu CPY, Cheung C, Wong JCH, Lienenkaëmper N, Cheung V, Suckling J, McAlonan GM (2008). Early striatal hypertrophy in first-episode psychosis within three weeks of initiating antipsychotic drug treatment. Psychological Medicine 39, 793-800.

Chua SE, Wright IC, Poline JB, Liddle PF, Murray RM, Frackowiak RS, Friston KJ, McGuire PK (1997). Grey matter correlates of syndromes in schizophrenia. A semi-automated analysis of structural magnetic resonance images. British Journal of Psychiatry 170, 406-410.

Crow TJ (1985). The two-syndrome concept, origins and current status. Schizophrenia Bulletin 11, 471-486.

Deng MY, McAlonan GM, Cheung C, Chiu CPY, Law CW, Cheung V, Sham PC, Chen EYH, Chua SE (2009). A naturalistic study of grey matter volume increase after early treatment in anti-psychotic naive, newly diagnosed schizophrenia. Psychopharmacology 206, 437-446.

Deutsch GK, Dougherty RF, Bammer R, Siok WT, Gabrieli JD, Wandell B (2005). Children's reading performance is correlated with white matter structure measured by diffusion tensor imaging. Cortex 41, 354-363.

Ellison-Wright I, Bullmore E (2009). Meta-analysis of diffusion tensor imaging studies in schizophrenia. Schizophrenia Research 108, 3-10.

Flaum M, O'Leary DS, Swayze VW, Miller DD, Arndt S, Andreasen NC (1995). Symptom dimensions and brain morphology in schizophrenia and related psychotic disorders. Journal of Psychiatry Research 29, 261-276.

Foong J, Maier M, Clark CA, Barker GJ, Miller DH, Ron MA (2000). Neuropathological abnormalities of the 
corpus callosum in schizophrenia, a diffusion tensor imaging study. Journal of Neurology Neurosurgery and Psychiatry 68, 242-244.

Foong J, Symms MR, Barker GJ, Maier M, Miller DH, Ron MA (2002). Investigating regional white matter in schizophrenia using diffusion tensor imaging. Neuroreport 13, 333-336.

Frankle WG, Lerma J, Laruelle M (2003). The synaptic hypothesis of schizophrenia. Neuron 39, 205-216.

Friedman JI, Tang C, Carpenter D, Buchsbaum M, Schmeidler J, Flanagan L, Golembo S, Kanellopoulou I, Ng J, Hof PR, Harvey PD, Tsopelas ND, Stewart D, Davis KL (2008). Diffusion tensor imaging findings in first-episode and chronic schizophrenia patients. American Journal of Psychiatry 165, 1024-1032.

Fujiwara H, Namiki C, Hirao K, Miyata J, Shimizu M, Fukuyama H, Sawamoto N, Hayashi T, Murai T (2007). Anterior and posterior cingulum abnormalities and their association with psychopathology in schizophrenia, a diffusion tensor imaging study. Schizophrenia Research 95, 215-222.

Genovese CR, Lazar NA, Nichols T (2002). Thresholding of statistical maps in functional neuroimaging using the false discovery rate. Neuroimage 15, 870-878.

Good CD, Johnsrude IS, Ashburner J, Henson RN, Friston KJ, Frackowiak RS (2001). A voxel-based morphometric study of ageing in 465 normal adult human brains. Neuroimage 14, 21-36.

Gupta RK, Saksena S, Agarwal A, Hasan KM, Husain M, Gupta V, Narayana PA (2005). Diffusion tensor imaging in late posttraumatic epilepsy. Epilepsia 46, 1465-1471.

Harrison PJ (1999). The neuropathology of schizophrenia. A critical review of the data and their interpretation. Brain 122, 593-624.

Horsfield MA, Larsson HB, Jones DK, Gass A (1998). Diffusion magnetic resonance imaging in multiple sclerosis. Journal of Neurology Neurosurgery and Psychiatry 64, S80-S84.

Hubl D, Koenig T, Strik W, Federspiel A, Kreis R, Boesch C, Maier SE, Schroth G, Lovblad K, Dierks T (2004). Pathways that make voices, white matter changes in auditory hallucinations. Archives of General Psychiatry 61, 658-668.

Kalus P, Buri C, Slotboom J, Gralla J, Remonda L, Dierks T, Strik WK, Schroth G, Kiefer C (2004). Volumetry and diffusion tensor imaging of hippocampal subregions in schizophrenia. Neuroreport 15, 867-871.

Kalus P, Slotboom J, Gallinat J, Federspiel A, Gralla J, Remonda L, Strik WK, Schroth G, Kiefer C (2005). New evidence for involvement of the entorhinal region in schizophrenia, a combined MRI volumetric and DTI study. Neuroimage 24, 1122-1129.

Kanaan R, Barker G, Brammer M, Giampietro V, Shergill S, Woolley J, Picchioni M, Toulopoulou T, McGuire P (2009). White matter microstructure in schizophrenia, effects of disorder, duration and medication. British Journal of Psychiatry 194, 236-242.

Kanaan RA, Kim JS, Kaufmann WE, Pearlson GD, Barker GJ, McGuire PK (2005). Diffusion tensor imaging in schizophrenia. Biological Psychiatry 58, 921-929.
Kay SR (1990). Positive-negative symptom assessment in schizophrenia, psychometric issues and scale comparison. Psychiatry Quarterly 61, 163-178.

Kay SR, Fiszbein A, Opler LA (1987). The positive and negative syndrome scale (PANSS) for schizophrenia. Schizophrenia Bulletin 13, 261-276.

Kay SR, Opler LA, Lindenmayer JP (1988). Reliability and validity of the positive and negative syndrome scale for schizophrenics. Psychiatry Research 23, 99-110.

Kubicki M, McCarley R, Westin CF, Park HJ, Maier S, Kikinis R, Jolesz FA, Shenton ME (2007). A review of diffusion tensor imaging studies in schizophrenia. Journal of Psychiatric Research 41, 15-30.

Kubicki M, Park H, Westin CF, Nestor PG, Mulkern RV, Maier SE, Niznikiewicz M, Connor EE, Levitt JJ, Frumin M, Kikinis R, Jolesz FA, McCarley RW, Shenton ME (2005). DTI and MTR abnormalities in schizophrenia, analysis of white matter integrity. Neuroimage 26, 1109-1118.

Kubicki M, Westin CF, Nestor PG, Wible CG, Frumin M, Maier SE, Kikinis R, Jolesz FA, McCarley RW, Shenton ME (2003). Cingulate fasciculus integrity disruption in schizophrenia, a magnetic resonance diffusion tensor imaging study. Biological Psychiatry 54, 1171-1180.

Kyriakopoulos M, Bargiotas T, Barker GJ, Frangou S (2008). Diffusion tensor imaging in schizophrenia. European Psychiatry 23, 255-273.

Kyriakopoulos M, Frangou S (2009). Recent diffusion tensor imaging findings in early stages of schizophrenia. Current Opinion in Psychiatry 22, 168-176.

Lappin LM, Morgan K, Morgan C, Hutchison G, Chitnis X, Suckling J, Fearon P, McGuire PK, Jones PB, Leff J, Murray RM, Dazzan P (2006). Gray matter abnormalities associated with duration of untreated psychosis. Schizophrenia Research 83, 145-153.

Leung MK, Cheung C, Yu K, Yip B, Sham PC, Li Q, Chua SE, McAlonan GM (2009). Gray matter in first-episode schizophrenia before and after antipsychotic drug treatment. Anatomical likelihood estimation meta-analyses with sample size weighting. Schizophrenia Bulletin. Published online: 16 September 2009. doi:10.1093/schbul/sbp099.

Leung LH, Ooi GC, Kwong DL, Chan GC, Cao G, Khong PL (2004). White-matter diffusion anisotropy after chemoirradiation, a statistical parametric mapping study and histogram analysis. Neuroimage 21, 261-268.

Lieberman J, Jody D, Geisler S, Alvir J, Loebel A, Szymanski S, Woerner M, Borenstein M (1993). Time course and biologic correlates of treatment response in first-episode schizophrenia. Archives of General Psychiatry 50, 369-376.

McAlonan GM, Cheung V, Cheung C, Suckling J, Lam GY, Tai KS, Yip L, Murphy DGM, Chua SE (2005). Mapping the brain in autism. A voxel-based MRI study of volumetric differences and intercorrelations in autism. Brain 128, 268-276.

Moseley M (2002). Diffusion tensor imaging and aging - a review. NMR Biomedicine 15, 553-560.

Narr KL, Toga AW, Szeszko P, Thompson PM, Wood RP, Robinson D, Sevy S, Wang Y, Schrock K, Bilder RM 
(2005). Cortical thinning in cingulate and occipital cortices in first episode schizophrenia. Biological Psychiatry $58,32-40$.

Okugawa G, Nobuhara K, Minami T, Tamagaki C, Takase K, Sugimoto T, Sawada S, Kinoshita T (2004). Subtle disruption of the middle cerebellar peduncles in patients with schizophrenia. Neuropsychobiology 50, 119-123.

Peters BD, de Haan L, Dekker N, Blaas J, Becker HE, Dingemans PM, Akkerman EM, Majoie CB, van Amelsvoort T, den Heeten GJ, Linszen DH (2008). White matter fibertracking in first-episode schizophrenia, schizoaffective patients and subjects at ultra-high risk of psychosis. Neuropsychobiology 58, 19-28.

Price G, Cercignani M, Parker GJ, Altmann DR, Barnes TR, Barker GJ, Joyce EM, Ron MA (2007a). Abnormal brain connectivity in first-episode psychosis, a diffusion MRI tractography study of the corpus callosum. Neuroimage 35, 458-466.

Price G, Cercignani M, Parker GJ, Altmann DR, Barnes TR, Barker GJ, Joyce EM, Ron MA (2007b). White matter tracts in first-episode psychosis, a DTI tractography study of the uncinate fasciculus. Neuroimage 39, 949-955.

Rapoport JL, Addington AM, Frangou S, Psych MR (2005). The neurodevelopmental model of schizophrenia, update 2005. Molecular Psychiatry 10, 434-449.

Schmithorst VJ, Wilke M, Dardzinski BJ, Holland SK (2005). Cognitive functions correlate with white matter architecture in a normal pediatric population, a diffusion tensor MRI study. Human Brain Mapping 26, 139-147.

Seok JH, Park HJ, Chun JW, Lee SK, Cho HS, Kwon JS, Kim JJ (2007). White matter abnormalities associated with auditory hallucinations in schizophrenia, a combined study of voxel-based analyses of diffusion tensor imaging and structural magnetic resonance imaging. Psychiatry Research 156, 93-104.

Shergill SS, Kanaan RA, Chitnis XA, O'Daly O, Jones DK, Frangou S, Williams SC, Howard RJ, Barker GJ, Murray RM, McGuire P (2007). A diffusion tensor imaging study of fasciculi in schizophrenia. American Journal of Psychiatry 164, 467-473.

Sigmundsson T, Suckling J, Maier M, Williams S, Bullmore E, Greenwood K, Fukuda R, Ron M, Toone B (2001). Structural abnormalities in frontal, temporal, and limbic regions and interconnecting white matter tracts in schizophrenic patients with prominent negative symptoms. American Journal of Psychiatry 158, 234-243.

Sim K, Yang GL, Loh D, Poon LY, Sitoh YY, Verma S, Keefe R, Collinson S, Chong SA, Heckers S, Nowinski W, Pantelis C (2009). White matter abnormalities and neurocognitive deficits associated with the passivity phenomenon in schizophrenia, a diffusion tensor imaging study. Psychiatry Research 172, 121-127.

Skelly LR, Calhoun V, Meda SA, Kim J, Mathalon DH, Pearlson GD (2008). Diffusion tensor imaging in schizophrenia, relationship to symptoms. Schizophrenia Research 98, 157-162.
Sowell ER, Thompson PM, Holmes CJ, Jernigan TL, Toga AW (1999). In vivo evidence for post-adolescent brain maturation in frontal and striatal regions. Nature Neuroscience 2, 859-861.

Sumich A, Chitnis XA, Fannon DG, O'Ceallaigh S, Doku VC, Faldrowicz A, Sharma T (2005). Unreality symptoms and volumetric measures of Heschl's gyrus and planum temporal in first-episode psychosis. Biological Psychiatry 57, 947-950.

Sun Z, Wang F, Cui L, Breeze J, Du X, Wang X, Cong Z, Zhang H, Li B, Hong N, Zhang D (2003). Abnormal anterior cingulum in patients with schizophrenia, a diffusion tensor imaging study. Neuroreport 14, 1833-1836.

Szeszko PR, Ardekani BA, Ashtari M, Kumra S, Robinson DG, Sevy S, Gunduz-Bruce H, Malhotra AK, Kane JM, Bilder RM, Lim KO (2005). White matter abnormalities in first-episode schizophrenia or schizoaffective disorder, a diffusion tensor imaging study. American Journal of Psychiatry 162, 602-605.

Szeszko PR, Robinson DG, Ashtari M, Vogel J, Betensky J, Sevy S, Ardekani BA, Lencz T, Malhotra AK, McCormack J, Miller R, Lim KO, Gunduz-Bruce H, Kane JM, Bilder RM (2008). Clinical and neuropsychological correlates of white matter abnormalities in recent onset schizophrenia. Neuropsychopharmacology 33, 976-984.

Thompson PM, Vidal C, Giedd JN, Gochman P, Blumental J, Nicholson R, Toga AW, Rappaport JL (2001). Mapping adolescent brain change reveals dynamic wave of accelerated gray matter loss in very early-onset schizophrenia. Proceedings of the National Academy of Sciences USA 98, 11650-11655.

Whitefield-Gabrieli S, Thermenos HW, Milanovic S, Tsuang MT, Faraone SV, McCarley RW, Shenton ME, Green AI, Nieto-Castanon A, LaViolette P, Wojcik J, Gabrieli JDE, Seidman LJ (2009). Hyperactivity and hyperconnectivity of the default network in schizophrenia and in first-degree relatives of persons with schizophrenia. Proceedings of the National Academy of Sciences USA 106, 1279-1284.

Wible CG, Anderson J, Shenton ME, Kricun A, Hirayasu Y, Tanaka S, Levitt JJ, O'Donnell BF, Kikinis R, Jolesz FA, McCarley RW (2001). Prefrontal cortex, negative symptoms, and schizophrenia, an MRI study. Psychiatry Research 108, 65-78.

Winterer G, Weinberger DR (2004). Genes, dopamine and cortical signal-to-noise ratio in schizophrenia. Trends in Neurosciences 27, 683-690.

Zetzsche T, Preuss U, Frodl T, Watz D, Schmitt G, Koutsouleris N, Born C, Reiser M, Möller H, Meisenzahl EM (2007). In-vivo topography of structural alterations of the anterior cingulate in patients with schizophrenia. New findings and comparison with the literature. Schizophrenia Research 96, 34-45. 